\title{
THE PERFORMANCE AND ROBUST IMPLEMENTATION OF A BLIND CMOE RECEIVER FOR MC-CDMA SYSTEMS
}

\author{
H. Cheng and S. C. Chan \\ Dept. of Electrical \& Electronic Engineering \\ The University of Hong Kong, Pokfulam Road, Hong Kong
}

\begin{abstract}
This paper studies a new robust constrained minimum output energy (CMOE)-based blind detector for multicarrier code division multiple access (MC-CDMA) systems. The proposed receiver intends to minimize the receiver's output energy while preserving the components of the desired user. By imposing quadratic weight constraints, the CMOE detector is made more robust against signal modeling errors, and a better performance over the standard CMOE detector is obtained. Steady-state signal-to-interference-plus-noise ratio (SINR) analysis of the blind CMOE detector and the minimum mean-squared error (MMSE) method with training sequences is presented. Numerical results show that the performance of the new robust detector is comparable to subspace-based method using exact singular value decomposition (SVD).
\end{abstract}

\section{INTRODUCTION}

MC-CDMA was recently proposed as an efficient multicarrier transmission scheme for supporting multiple access communications [1]. It combines CDMA and orthogonal frequency division multiplexing (OFDM) techniques. It has received considerable attention because of its advantages in frequency diversity, multipath fading resilience and etc [2].

Similar to conventional CDMA systems, MC-CDMA is an interference-limited system. It is rather sensitive to multiple access interference (MAI), which arises from the other users signals transmitted using the same frequency band. In [3], Honig et al proposed a blind linear MMSE receiver, called the CMOE detector, for MAI suppression in CDMA systems. The CMOE detector minimizes the receiver's output energy while preserving the components of the desired user in the received signal. Under ideal conditions, it has a performance close to the exact MMSE solution [4]. Also, it can be conveniently implemented using the standard RLS algorithm of $O\left(M^{2}\right)$ computational complexity ( $M$ is the length of the spreading code).

However, it is found that the performance of the CMOE detector will considerably degrade due to channel estimation error and other possible types of signal mismatch [5] [6]. In this paper, a robust $\mathrm{CMOE}$ detector with quadratic weight constraint is proposed for MC-CDMA systems. This technique is a simple and effective method for improving the robustness of the detector with respect to signal modeling errors. A CMOE-based channel estimator is also presented, where the channel can be estimated blindly by solving the minimum eigenvector of a matrix. Subspace-based blind MMSE receivers [7] are also investigated to compare the performance of the CMOE-based and subspace-based methods. The performances of the subspacebased MMSE detector using the exact SVD and the robust CMOE detector are found to be comparable.

The rest of the paper is organized as follows: Section 2 describes the principle of MC-CDMA systems. Section 3 is devoted to the robust CMOE receiver, while SINR analysis is presented in Section 4. Section 5 gives the numerical examples, and finally, conclusion is drawn in Section 6.

\section{SYSTEM MODEL}

We consider a MC-CDMA system for uplink transmission from the terminal users to the base station. Fig. 1 shows the structure of an MC-CDMA transmitter. The original data stream of the $k$ th user is first converted into $P$ parallel data sequences. Each serial/ parallel $(\mathrm{S} / \mathrm{P})$ converted output spreads with a spreading sequence of length $M$. The data chips after spreading are $\mathrm{S} / \mathrm{P}$ converted into $M$ parallel subcarriers. The total $N=P M$ chips are modulated by the IDFT and converted back into serial data. The cyclic prefix is inserted between symbols to avoid intersymbol interference caused by multipath fading. Let $T$ be the symbol duration of the original data stream, and $T_{S}$ be the one at the subcarrier, then it has $T_{S}=P T$. The frequency separation between the successive subcarriers is $\Delta f=1 / T_{S}$. The complex equivalent transmitted signal of user $k$ can be written as

$$
s_{k}(t)=\sum_{i=-\infty}^{i} \sqrt{P_{k}} \sum_{p=0}^{P-1} \sum_{m=0}^{M-1} b_{k, p}^{(i)} c_{k, m}^{(i)} \psi\left(t-i T_{s}\right) e^{j 2 \pi(P m+p) \Delta f t},
$$

where $P_{k}$ is the chip energy, $b_{k, p}^{(i)}$ is the transmitted $i t h$ data bit in the $p t h$ data stream, $c_{k, m}^{(i)}$ is the $m t h$ chip of the given spreading sequence, and $\psi(t)$ represents the rectangular pulse waveform. Note that there are $P$ data streams for the $k t h$ user, and the data bits on different streams use the same spreading sequence. In order to achieve the maximum frequency diversity, the subcarrier separation for $b_{k, p}^{(n)}$ is $\Delta f^{\prime}=P / T_{s}$. The kth user's signal $s_{k}(t)$ propagates through a frequency-selective Rayleigh fading channel with $L$ received paths. The channel is represented by a time-variant impulse response

$$
g_{k}(t)=\sum_{l=1}^{L} \alpha_{k, l}(t) \delta\left(t-\tau_{k, l}\right),
$$


where $k$ is the user index, $\alpha_{k, l}(t)$ is the lth path gain which is independent zero mean, complex Gaussian random process, and $\tau_{k, l}$ is the propagation delay for the lth path, respectively.

In MC-CDMA systems, because of the large symbol duration, each subcarrier approximately experiences flat fading. Assume that there are $K$ simultaneous users in the system, all using the same selection of $P$ and $M$. At the receiver, the signal is sampled at a rate $N / T_{S}$, the samples corresponding to the cyclic prefix are discarded, and a DFT of size $N$ is performed. For simplicity, we will consider a synchronous model, where all the users arrive at the same time. The received signal $\mathbf{y}_{p}(i)=\left[y_{p}(i), \cdots, y_{P \cdot(M-1)+p}(i)\right]^{T}$ after DFT operation with respect to the data symbol $b_{k, p}^{(i)}$ can be given as

$$
\mathbf{y}_{p}(i)=\sqrt{P_{k}} \sum_{k=0}^{K-1} \mathbf{C}_{k} \mathbf{h}_{k, p}^{(i)} b_{k, p}^{(i)}+\mathbf{n}(i)=\sum_{k=0}^{K-1} \sqrt{P_{k}} \mathbf{d}_{k, p}^{(i)} b_{k, p_{k}}^{(i)}+\mathbf{n}(i),
$$

where (for convenience, the subscript $i$ is omitted) $b_{k, p}$ is the data symbol corresponding to the $p t h$ data stream; $P_{k}$ is the chip energy; $\mathbf{h}_{k, p}$ denotes channel frequency response, whose element is $h_{k, P m+p}=\frac{1}{\sqrt{N}}\left(\sum_{l=0}^{L-1} \alpha_{k, l} e^{-\frac{j 2 \pi l(P m+p)}{N}}\right), m=0, \ldots, M-1 ; \mathbf{C}_{k}=$ $\operatorname{diag}\left\{c_{k}[0], c_{k}[1], \ldots, c_{k}[M-1]\right\}$ is the code matrix; $\mathbf{d}_{k, p}=\left[c_{k}^{0} h_{k, p}\right.$, $\left.\cdots, c_{k}^{M-1} h_{k, P(M-1)+p}\right]^{T}$ represents the effective signature; and $\mathbf{n}=\left[\eta_{p}, \eta_{P+p}, \cdots, \eta_{P(M-1)+p}\right]^{T}$ denotes complex additive white Gaussian with zero mean and variance $\sigma_{v}^{2}$.

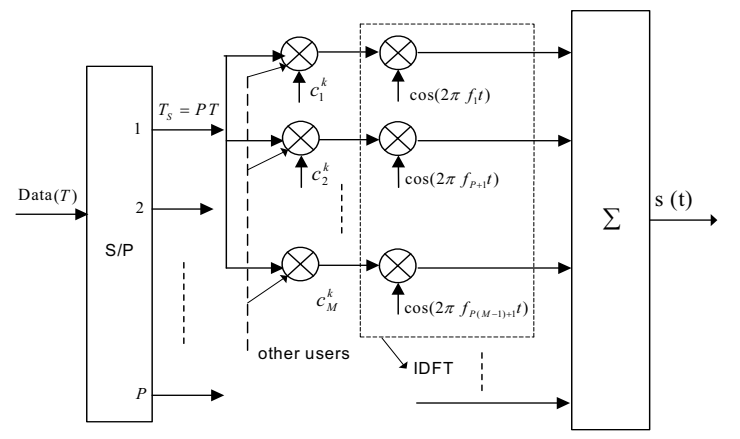

Fig. 1. Transmitter structure for MC-CDMA.

\section{ROBUST CMOE DETECTION}

In practical applications, the estimated effective signature waveform $\tilde{\mathbf{d}}_{0}$ generally contains errors. In addition, the covariance matrix is estimated from a finite number of the observed data. The performance of the standard CMOE detector will degrade greatly due to these two advert factors [3] [5]. Robust CMOE detectors are thus desirable to overcome the signal modeling errors. One simple and efficient method is to impose quadratic constraints on the norm of the weight vector $\mathbf{w}(n)$. Without loss of generality, the subscript $p$ will be omitted from Eq. (3), and user 1 is the desired user. The CMOE detector with quadratic constraints then can be formulated as follows

$$
\min _{\mathbf{w}} \sum_{i=1}^{n} \lambda^{n-i}\left|\mathbf{w}^{H}(n) \mathbf{y}(i)\right|^{2}, \text { s.t. } \mathbf{w}^{H}(n) \mathbf{d}_{0}=1, \mathbf{w}^{H} \mathbf{w} \leq T_{0},
$$

where $0<\lambda<1$ is the forgetting factor, and $\mathbf{d}_{0}$ is the effective signature waveform of the desired user. To meet $\mathbf{w}^{H}(n) \mathbf{d}_{0}=1$, a vector $\mathbf{w}_{q}$ is defined as $\mathbf{w}_{q}=\mathbf{d}_{0}\left(\mathbf{d}_{0}^{H} \mathbf{d}_{0}\right)^{-1}$. A reasonable constraint value is $T_{0}=2\left\|\mathbf{w}_{q}\right\|^{2}$. The solution to the constrained optimization problem in Eq. (4) is given by

$$
\begin{aligned}
\mathbf{w} & =\left[\mathbf{d}_{0}^{H}\left(\mathbf{R}_{\mathbf{y}}+\beta \mathbf{I}\right)^{-1} \mathbf{d}_{0}\right]^{-1}\left(\mathbf{R}_{\mathbf{y}}+\beta \mathbf{I}\right)^{-1} \mathbf{d}_{0} \\
& =\left[\left(\mathbf{I}+\beta \mathbf{R}_{\mathbf{y}}^{-1}\right)^{-1} \mathbf{R}_{\mathbf{y}}^{-1} \mathbf{d}_{0}\right] /\left[\mathbf{d}_{0}^{H}\left(\mathbf{I}+\beta \mathbf{R}_{\mathbf{y}}^{-1}\right)^{-1} \mathbf{R}_{\mathbf{y}}^{-1} \mathbf{d}_{0}\right] .
\end{aligned}
$$

Inspired by the approach in [9], we propose an algorithm to compute the diagonal loading $\beta$ given a quadratic constraint $T_{0}$, and to implement RLS updating for Eq. (5). For small $\beta$, the term $\left(\mathbf{I}+\beta \mathbf{R}_{\mathbf{y}}^{-1}\right)^{-1}$ can be approximated using the first two terms of its Taylor series expansion, i.e., $\left(\mathbf{I}+\beta \mathbf{R}_{\mathbf{y}}^{-1}\right)^{-1} \approx \mathbf{I}-\beta \mathbf{R}_{\mathbf{y}}^{-1}$. With this approximation, Eq. (5) becomes

$$
\mathbf{w} \approx\left(\mathbf{R}_{\mathbf{y}}^{-1} \mathbf{d}_{0}-\beta \mathbf{R}_{\mathbf{y}}^{-1} \mathbf{R}_{\mathbf{y}}^{-1} \mathbf{d}_{0}\right) /\left(\mathbf{d}_{0}^{H} \mathbf{R}_{\mathbf{y}}^{-1} \mathbf{d}_{0}-\beta \mathbf{d}_{0}^{H} \mathbf{R}_{\mathbf{y}}^{-1} \mathbf{R}_{\mathbf{y}}^{-1} \mathbf{d}_{0}\right) .
$$

Let $r=\mathbf{d}_{0}^{H} \mathbf{R}_{\mathbf{y}}^{-1} \mathbf{d}_{0}, \quad \mathbf{v}=\mathbf{R}_{\mathbf{y}}^{-1} \mathbf{R}_{\mathbf{y}}^{-1} \mathbf{d}_{0}=r \mathbf{R}_{\mathbf{y}}^{-1} \widetilde{\mathbf{w}}$, and $\widetilde{\mathbf{w}}=\mathbf{R}_{\mathbf{y}}^{-1} \mathbf{d}_{0} / r$. We get $\mathbf{w} \approx(\widetilde{\mathbf{w}}-\beta \mathbf{v}) /\left(1-r \beta \cdot \widetilde{\mathbf{w}}^{H} \widetilde{\mathbf{w}}\right)$. When $\widetilde{\mathbf{w}}$ does not satisfy the norm constraint, we can solve the following equality for $\beta$,

$$
\mathbf{w}^{H} \mathbf{w}-T_{0}=a \beta^{2}+b \beta+c=0,
$$

where $\quad a=T_{0} r^{2}\|\widetilde{\mathbf{w}}\|^{4}-\|\mathbf{v}\|^{2}, \quad b=2\left[\Re e\left(\widetilde{\mathbf{w}}^{H} \mathbf{v}-r T_{0}\|\widetilde{\mathbf{w}}\|^{2}\right], \quad\right.$ and $c=T_{0}-\|\widetilde{\mathbf{w}}\|^{2}$. The solution of the diagonal loading $\beta$ should be real, non-negative value. More details of choosing the value of $\beta$ from Eq. (7) are presented in [9]. An appropriate solution of Eq. (7) is $\beta=\left(-b-\mathfrak{R} e\left(\sqrt{b^{2}-4 a c}\right) /(2 a)\right.$.

A RLS algorithm for updating the weight vector $\mathbf{w}(n)$ in Eq. (5) can be summarized as follows:

$$
\begin{aligned}
& \text { Initiation }: \mathbf{w}(0)=\mathbf{0}, \mathbf{P}[0]=\delta^{-1} \mathbf{I}_{M \times M} ; \\
& \text { Update }: \mathbf{K}[n]=\frac{\mathbf{P}[n-1] \mathbf{y}[n]}{\lambda+\mathbf{y}^{H}[n] \mathbf{P}[n-1] \mathbf{y}[n]}, \\
& \mathbf{P}[n]=\frac{1}{\lambda}\left\{\mathbf{P}[n-1]-\mathbf{K}[n] *^{*} \mathbf{y}^{H}[n] \mathbf{P}[n-1]\right\}, \\
& \mathbf{w}[n]=\left(\mathbf{d}_{0}^{H} \mathbf{P}[n] \mathbf{d}_{0}\right)^{-1} \mathbf{P}[n] \mathbf{d}_{0}, \mathbf{w}_{q}[n]=\mathbf{d}_{0}\left(\mathbf{d}_{0}^{H} \mathbf{d}_{0}\right)^{-1}, T_{0}=\left\|\mathbf{w}_{q}[n]\right\|^{2}, \\
& \text { if } \quad\|\mathbf{w}[n]\|^{2}>T_{0}, \quad r=\left(\mathbf{d}_{0}^{H} \mathbf{P}[n] \mathbf{d}_{0}\right), \mathbf{v}[n]=r \mathbf{P}[n] \mathbf{w}[n], \\
& a=T_{0} r^{2}\|\mathbf{w}[n]\|^{4}-\|\mathbf{v}[n]\|^{2}, b=2[\Re e(\mathbf{w}[n] \\
& c=T_{0}-\|\mathbf{w}[n]\|^{2}, \beta=\left[-b-\Re T_{0}\|\mathbf{w}[n]\|^{2}\right], \\
& \left.\mathbf{w}[n]=(\mathbf{w}[n]-\beta \mathbf{v}[n]) /\left(1-r \beta \cdot \mathbf{w}[n] b^{H}-4 a c\right)\right] / 2 a,
\end{aligned}
$$

Next, we consider a CMOE-based channel estimator. For a standard CMOE detector, the ideal solution is give by [3]

$$
\mathbf{w}_{o}=\left(\mathbf{d}_{0}^{H} \mathbf{R}_{y}^{-1}(n) \mathbf{d}_{0}\right)^{-1} \mathbf{R}_{y}^{-1}(n) \mathbf{d}_{0} .
$$

The resulting minimum output variance of the $\mathrm{CMOE}$ detector is 


$$
P_{\min }=\mathbf{w}_{o}^{H} \mathbf{R}_{y}(n) \mathbf{w}_{o}=\left(\mathbf{d}_{0}^{H} \mathbf{R}_{y}^{-1}(n) \mathbf{d}_{0}\right)^{-1} .
$$

We can minimize the reciprocal of Eq. (9) with respect to $\mathbf{d}_{0}$ to maximize the signal components at the receiver output,

$$
\hat{\mathbf{d}}_{0}=\underset{\mathbf{d}_{0}}{\arg \min } \mathbf{d}_{0}^{H} \mathbf{R}_{y}^{-1}(n) \mathbf{d}_{0} .
$$

Consider a channel with $L$ multipaths, and $\mathbf{g}_{0}$ is the channel impulse response. It has $\mathbf{h}_{0}=\mathbf{F}_{m} \mathbf{g}_{0}$, where $\mathbf{F}_{m} \in \mathbf{C}^{M \times L}$ is a DFT matrix. We then have $\mathbf{d}_{0}=\mathbf{C}_{0} \mathbf{F}_{m} \hat{\mathbf{g}}_{0}$, where $\mathbf{C}_{0}$ is the code matrix of the desire user, and $\hat{\mathbf{g}}_{0}$ denotes the estimation of $\mathbf{g}_{0}$. Therefore, minimizing Eq. (10) with respect to $\mathbf{g}_{0}$ yields

$$
\hat{\mathbf{g}}_{0}=\arg \min _{\hat{g}_{0} \in C^{L \times 1}} \mathbf{g}_{0}^{H}[\underbrace{\left[\mathbf{F}_{m}^{H} \mathbf{C}_{0}^{H} \mathbf{R}_{\mathbf{y}}^{-1}(n) \mathbf{C}_{0} \mathbf{F}_{m}\right]}_{\mathbf{\Omega}} \mathbf{g}_{0} .
$$

It can be seen that the channel estimate $\hat{\mathbf{g}}_{0}$ is the eigenvector associated with the smallest eigenvalue of the matrix $\boldsymbol{\Omega}$.

\section{SINR ANALYSIS}

In this section, the steady-state SINR of blind CMOE and training MMSE detectors both with RLS adaptation rule will be presented. Assume that the transmitting symbols among the users are zero mean and i.i.d. sequences. Consequently, we have $\mathbf{R}_{\mathbf{y}}=E\left\{\mathbf{y} \mathbf{y}^{H}\right\}=P_{0} \mathbf{d}_{0} \mathbf{d}_{0}^{H}+\left(\sum_{k=1}^{K-1} P_{k} \mathbf{d}_{k} \mathbf{d}_{k}^{H}+\sigma_{n}^{2} \mathbf{I}\right)=\mathbf{R}_{s}+\mathbf{R}_{\text {in }} \quad . \quad$ The output SINR is defined as $\operatorname{SINR}=\left(\mathbf{w}^{H} \mathbf{R}_{s} \mathbf{w}\right) /\left(\mathbf{w}^{H} \mathbf{R}_{\text {in }} \mathbf{w}\right)$. Under ideal conditions, the CMOE detector maximizes the output SINR, that is, $\operatorname{SINR}_{\text {opt }}=P_{0}\left(\mathbf{d}_{0}^{H} \mathbf{R}_{\text {in }}^{-1} \mathbf{d}_{0}\right)$.

\section{Steady-state SINR for RLS adaptive blind CMOE detector:}

The mean value $E\left\{\mathbf{w}(n){ }^{H} \mathbf{R}_{S} \mathbf{w}(n)\right\}$ is given as

$$
E\left\{\left|\mathbf{w}^{H}(n-1) \sqrt{P_{0}} \mathbf{d}_{0} b_{0}(n)\right|^{2}\right\}=P_{0} E\left\{\mathbf{w}^{H}(n-1) \mathbf{d}_{0}\right\}=P_{0} .
$$

Let the optimal weight vector be $\mathbf{w}_{o}$, and $\zeta(n)=\mathbf{w}(n)-\mathbf{w}_{o}$. Therefore, $\eta(n)=E\left\{\mathbf{w}(n)^{H} \mathbf{R}_{y} \mathbf{w}(n)\right\}$ is given by

$$
\begin{aligned}
& \eta(n)=E\left\{\left[\overline{\mathbf{w}}^{H}+\zeta^{H}(n-1)\right] \mathbf{y}(n) \mathbf{y}{ }^{H}(n)[\overline{\mathbf{w}}+\zeta(n-1)]\right\} \\
& =\bar{\eta}+\operatorname{tr}\left[\mathbf{R}_{\mathbf{y}} \mathbf{K}(n-1)\right]+2 \overline{\mathbf{w}} \mathbf{R}_{\mathbf{y}} E\{\zeta(n-1)\},
\end{aligned}
$$

where $\bar{\eta}=\overline{\mathbf{w}}^{H} \mathbf{R}_{\mathbf{y}} \overline{\mathbf{w}}=P_{0}\left(1+1 / \operatorname{SINR}_{\text {opt }}\right)$. Since $E\{\zeta(n)\} \rightarrow 0$ as $n \rightarrow \infty$, we have $\eta(n) \approx \bar{\eta}+\eta_{e x}(n)$. In [10], it derives $\left.\eta_{\text {ex }}(n)=\operatorname{tr}\left[\mathbf{R}_{\mathbf{y}} \mathbf{K}(n-1)\right]=\left[\lambda^{2}+(1-\lambda)^{2}\right] \eta_{e x}(n-1)\right]+(1-\lambda)^{2}(M-1) \bar{\eta}$, where $M$ is the length of $\mathbf{w}$. Since $\lambda^{2}+(1-\lambda)^{2}<1$ for $0 \leq \lambda \leq 1 \quad, \quad \eta_{\text {ex }}(n) \quad$ converges, that is, $\eta_{e x}(\infty)=\lim _{n \rightarrow \infty} \operatorname{tr}\left[\mathbf{R}_{\mathbf{y}} \mathbf{K}(n)\right]=v \bar{\eta}$, where $v=(1-\lambda)(M-1) /(2 \lambda)$. The steady-state SINR of the RLS adaptive CMOE detector can be obtained as

$$
\operatorname{SINR}_{\text {moe }}^{\infty}=\operatorname{SINR}_{\text {opt }} /\left[(1+v)+v \cdot \operatorname{SINR}_{\text {opt }}\right] .
$$

Steady-state SINR for RLS adaptive MMSE detector:
In this case, the exponentially windowed RLS algorithm chooses $\mathbf{w}(n)$ to minimize the cost function

$$
\sum_{i=1}^{n} \lambda^{n-i}\left|\sqrt{P_{0}} b_{0}(i)-\mathbf{w}^{H}(n) \mathbf{y}(i)\right|^{2} .
$$

As $n \rightarrow \infty, \mathbf{w}(n)$ converges to the optimal solution $\overline{\mathbf{w}}=$ $P_{0} \mathbf{R}_{\mathbf{y}}^{-1} \mathbf{d}_{0}$. Let $\bar{\zeta}(n)=\mathbf{w}(n)-\overline{\mathbf{w}}$. Then $\eta_{s}=E\left\{\mathbf{w}^{H}(n) \mathbf{R}_{s} \mathbf{w}(n)\right\}$ is

$$
\begin{aligned}
\eta_{S} & =E\left\{\left[\overline{\mathbf{w}}^{H}+\bar{\zeta}^{H}(n-1)\right] P_{0} \mathbf{d}_{0} \mathbf{d}_{0}^{H}[\overline{\mathbf{w}}+\bar{\zeta}(n-1)]\right\} \\
& =\overline{\mathbf{w}}^{H} \mathbf{R}_{s} \overline{\mathbf{w}}+\operatorname{tr}\left[\mathbf{R}_{s} \mathbf{K}(n-1)\right]+2 \overline{\mathbf{w}}^{H} \mathbf{R}_{s} E\{\bar{\zeta}(n-1)\},
\end{aligned}
$$

where $\overline{\mathbf{w}}^{H} \mathbf{R}_{s} \overline{\mathbf{w}}=P_{0}\left(P_{0} \mathbf{d}_{0}^{H} \mathbf{R}_{\mathbf{y}}^{-1} \mathbf{d}_{0}\right)^{2}$, and $E\{\bar{\zeta}(n)\} \rightarrow 0$ as $n \rightarrow \infty$. The weight error correlation matrix $\mathbf{K}(n)$ is given as [10]

$$
\overline{\mathbf{K}}(n)=E\left\{\bar{\zeta}(n) \bar{\zeta}^{H}(n)\right\} \approx \lambda^{2} \overline{\mathbf{K}}(n-1)+(1-\lambda)^{2} \mathbf{R}_{\mathbf{y}}^{-1} \bar{\varepsilon} .
$$

where $\bar{\varepsilon}$ is the MSE of the optimum filter $\overline{\mathbf{w}}$, given by

$$
\bar{\varepsilon}=E\left\{\left|\sqrt{P_{0}} b_{0}-\overline{\mathbf{w}}^{H} \mathbf{y}\right|^{2}\right\}=\frac{P_{0}}{1+P_{0}\left(\mathbf{d}_{0}^{H} \mathbf{R}_{\text {in }}^{-1} \mathbf{d}_{0}\right)}=\frac{P_{0}}{1+\operatorname{SINR}_{\text {opt }}} .
$$

Premultiplying both sides of Eq. (17) by $\mathbf{R}_{s}$, then taking the trace, it yields $\operatorname{tr}\left[\mathbf{R}_{s} \mathbf{K}(n)\right] \approx \lambda^{2} \operatorname{tr}\left[\mathbf{R}_{s} \mathbf{K}(n-1)\right]+(1-\lambda)^{2} P_{0}\left(\mathbf{d}_{0}^{H} \mathbf{R}_{\mathbf{y}}^{-1} \mathbf{d}_{0}\right) \bar{\varepsilon}$. Hence, $\operatorname{tr}\left[\mathbf{R}_{S} \mathbf{K}(n)\right]$ converges for $0 \leq \lambda \leq 1$, and it has $\bar{\eta}_{e x}(\infty)=$ $\lim _{n \rightarrow \infty} \operatorname{tr}\left[\mathbf{R}_{S} \mathbf{K}(n)\right]=P_{0} \cdot \frac{1-\lambda}{1+\lambda} \cdot \frac{S I N R_{o p t}}{\left(1+S I N R_{o p t}\right)^{2}}$. In practical applications, $\lambda$ is around 0.9 . Therefore, with reasonably high SNR, it has $\bar{\eta}_{e x}(\infty) \rightarrow 0$, and $E\left\{\mathbf{w}^{H}(n) \mathbf{R}_{S} \mathbf{w}(n)\right\}$ can be approximated by

$$
E\left\{\mathbf{w}^{H}(n) \mathbf{R}_{S} \mathbf{w}(n)\right\} \approx \overline{\mathbf{w}}^{H} \mathbf{R}_{s} \overline{\mathbf{w}}=P_{0}\left(\frac{\text { SINR }_{\text {opt }}}{1+\operatorname{SINR}_{\text {opt }}}\right)^{2} .
$$

It is shown that [8] $\eta(n)=E\left\{\mathbf{w}^{H}(n) \mathbf{R}_{y} \mathbf{w}(n)\right\}=E\left\{\left|\mathbf{w}^{H}(n-1) \mathbf{y}(n)\right|^{2}\right\}$ $=P_{0}\left(v+\operatorname{SINR}_{\text {opt }}\right) /\left(1+\operatorname{SIN} R_{\text {opt }}\right)$. Therefore, the output SINR of the RLS adaptive MMSE detector is given by

$$
\operatorname{SINR}_{\text {mmse }}^{\infty}=\operatorname{SINR}_{\text {opt }} /\left[(1+v)+v / \operatorname{SINR}_{\text {opt }}\right] .
$$

The steady-state output SINR given in Eq. (14) and (19) is consistent to the formulations in [9], where the SINR is defined as $\operatorname{SINR}^{\infty}=\lim _{n \rightarrow \infty}\left\{E^{2}\left[\mathbf{w}^{H}(n-1) \mathbf{y}(n)\right]\right\} /\left\{\operatorname{var}\left[\mathbf{w}^{H}(n-1) \mathbf{y}(n)\right]\right\}$.

\section{NUMERICAL RESULTS}

Numerical simulations have been implemented to illustrate the performance of the proposed robust CMOE detector. We consider a synchronous MC-CDMA system in uplink channels with random spreading sequence. The quadratic constraint is set as $T_{0}=1.5\left\|\mathbf{w}_{q}\right\|^{2}$. SNR is defined as the average transmitted bit energy to noise ratio $P_{k} / \sigma_{v}^{2}$. The multiple access interferers (MAI's) are $10-\mathrm{dB}$, i.e., $P_{k} / P_{0}=10$. The scalar ambiguity of the blind channel estimator is compensated with a complex constant, which makes the first element of the estimation $\hat{\mathbf{h}}_{0, p}$ and that of the true channel vector $\mathbf{h}_{0, p}$ identical. 


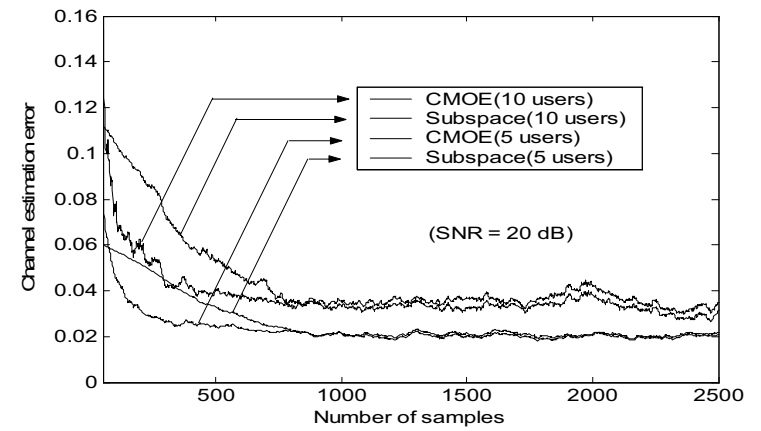

Fig. 2. Channel estimation error for the CMOE-based and subspace-based approaches. $S N R=20 d B, \lambda=0.995$, and $\delta=0.1$.

First, the steady-state performance of the detectors is investigated. We set $M=32$ and $P=3$. A multipath channel with six paths is considered. The channel coefficients are randomly generated complex values. The simulation results are averaged over 200 Monte-Carlo trials. Fig. 2 shows the channel estimation error of the CMOE-based and subspace-based channel estimators. The normalized channel estimation error is measured by $\left\|\mathbf{h}_{0, p}-\hat{\mathbf{h}}_{0, p}\right\|^{2} /\left\|\mathbf{h}_{0, p}\right\|^{2}$. To recursively implement the subspace-based channel estimation, QR inverse iteration noise subspace tracking algorithm [11] is adopted. It shows that, at high SNR, the CMOE-based channel estimator has a similar performance to the subspace-based method. Fig. 3 illustrates the output SINR of the different linear MMSE detectors versus input SNR. It can be seen that the SINR performance of the proposed robust $\mathrm{CMOE}$ detector is much better than the CMOE, and it is comparable to that of the subspace-based blind MMSE detector.

Next, a slowly time-varying channel with $L=6$ Rayleigh fading paths in indoor environments is considered, where the multipath intensity profile decays exponentially. The maximum Doppler frequency $\left(f_{D}\right)$ is $52 \mathrm{~Hz}$ corresponding to a terminal speed $v=3 \mathrm{~m} / \mathrm{s}$ with a carrier frequency of $5.2 \mathrm{GHz}$. The subcarrier spacing $(\Delta f)$ is $312.5 \mathrm{KHz}$, and the time duration at the subcarrier is $T_{S}=3.2 \mu \mathrm{s}$. Hence, the normalized fading rate is $f_{D} T_{S}=1.664 \times 10^{-4}$. We average simulation results over 500 Monte-Carlo trials. Fig. 4 depicts the BER comparison for the CMOE detectors and the subspace-based receiver. To save simulation time, we set $M=16$ and $P=8$. Again, it can be seen that the robust $\mathrm{CMOE}$ detector performs much better than the $\mathrm{CMOE}$ detector and the performance is comparable to the subspace-based method.

\section{CONCLUSIONS}

In this paper, a robust CMOE blind detector for MC-CDMA systems is proposed. By imposing quadratic weight constraints, the CMOE detector is made more robust against signal modeling errors, and a better performance over the standard CMOE detector is obtained. A convenient adaptive implementation of the robust CMOE detector is presented. Steady-state SINR performance of the blind CMOE detector and the training MMSE method is also presented.

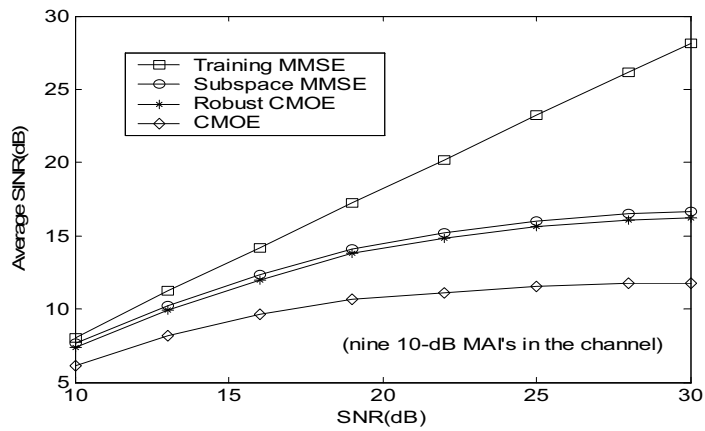

Fig. 3. Output SINR for the blind MMSE detectors and training MMSE versus SNR. $K=10, \lambda=0.995$, and $\delta=0.1$.

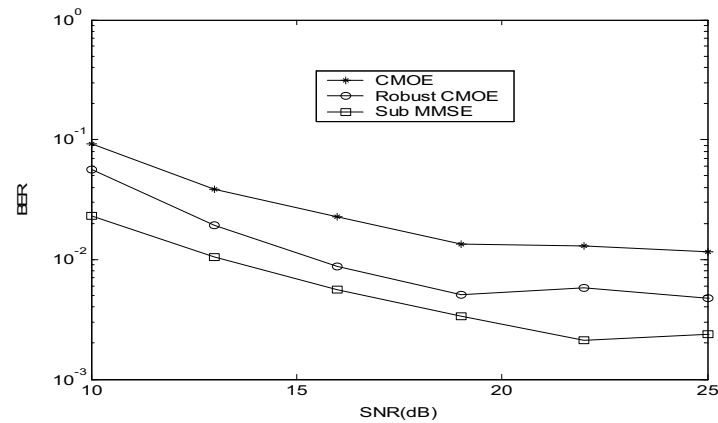

Fig. 4. BER comparison when $M=16, P=8, K=7$, six 10-dB MAI's in the channel, $\lambda=0.96$, and $\delta=0.1$.

\section{REFERENCES}

[1] N. Yee, J.P. Linnartz, and G. Fettweis, "Multicarrier CDMA in indoor wireless radio networks", PIMRC, pp. 109-133, vol. 1, 1993.

[2] S. Hara and R. Prasad, "Overview of multicarrier CDMA", IEEE Commun. Magazine, vol. 35, pp. 126-133, Dec. 1997.

[3] M. Honig, U. Madhow and S. Verdu, "Blind multiuser detection", IEEE Trans. Inform. Theory, vol. 41, pp. 944-960, July 1995.

[4] M. K. Tsatsanis and Z.Y. Xu, "Performance analysis of minimum variance CDMA receivers", IEEE Trans. Signal Processing, Vol. 46, No. 11, Nov. 1998.

[5] H. Cox, R. Zeskind, and M. Owen, "Robust adaptive beamforming", IEEE Trans. ASSP, vol. 35, pp. 1365-1376, 1987.

[6] Z. Tian, K. L. Bell, and H.L. Van Trees, "Robust constrained linear receivers for CDMA wireless systems", IEEE Trans. Commun., vol. 49, pp. 1510-1522, July 2001.

[7] J. Namgoong, T.F. Wong and J.S. Lehnert, "Subspace MMSE receiver for multicarrier CDMA", IEEE WCNC '99, pp. 90-94, New Orleans, LA, 1999.

[8] Z. Tian, K. L. Bell, and H.L. Van Trees, "A recursive least squares implementation for LCMP beamforming under quadratic constraint”, IEEE Trans. Signal Processing, vol. 49, pp. 113811145 , June 2001

[9] H.V. Poor and X.D. Wang, "Code-aided interference suppression for DS/CDMA communications-part II: parallel blind adaptive implementations", IEEE Trans. Commun., Vol. 45, No. 9, 1997.

[10] E. Eleftheriou and D. D. Falconer, "Tracking properties and steadystate performance of RLS adaptive filter algorithms", IEEE Trans. ASSP, vol. 34, pp. 1097-1109, Oct. 1986.

[11] P. Strobach, "Square-root QR inverse iteration for tracking the minor subsapce", IEEE Trans. Signal Processing, vol. 48, pp. 29942999, Nov. 2000. 